\title{
Fièvre récurrente chez un voyageur de retour du Sénégal
}

\author{
Eric J. Eckbo MD, Marthe Charles MD MSc, Robert Wolber MD, Gannon Yu MD
}

- Citation : CMAJ 2021 February 22;193:E285-8. doi : 10.1503/cmaj.201644-f

Voir la version anglaise de l'article ici : www.cmaj.ca/lookup/doi/10.1503/cmaj.201644

$\mathbf{U}$

n homme de 24 ans a consulté son médecin de famille parce qu'il présentait depuis la veille des symptômes subjectifs de fièvre, des frissons, une céphalée, une myalgie, une arthralgie et une diarrhée légère; le médecin lui a conseillé de se rendre au service des urgences pour des examens plus approfondis. À son arrivée dans notre service d'urgence de la région de Vancouver, sa température était de $38,3^{\circ} \mathrm{C}$ (température maximale au dossier $38,4^{\circ} \mathrm{C}$ ), sa tension artérielle de $117 / 67 \mathrm{~mm} \mathrm{Hg}$, sa fréquence cardiaque de 88 battements/ minute, et sa fréquence respiratoire de 16 respirations/minute. II était en diaphorèse, mais semblait bien pour le reste.

Ce patient arrivait d'un long voyage; il était de retour depuis 9 jours. Huit mois auparavant, il était parti seul pour l'Europe, puis s'était rendu au Maroc avant de passer quelques semaines au Sénégal. Il n'avait reçu aucune recommandation médicale ni vaccin en prévision de son voyage et n'avait pas pris d'antipaludéens prophylactiques. Au Sénégal, il a séjourné dans un petit village et logé chez l'habitant. Il a consommé l'eau et les aliments locaux. Il passait la majeure partie de son temps à découvrir la région à vélo. II n'a eu aucun contact sexuel ni côtoyé de gens malades. Le patient a remarqué qu'il y avait beaucoup de chiens dans ce village, et que beaucoup étaient infestés de tiques. Au milieu de son séjour, il s'est fait piquer à la jambe par ce qu'il croit être un insecte, et la lésion est devenue prurigineuse et rouge. Il a ensuite présenté des symptômes subjectifs de fièvre, frissons et céphalées pendant 4 jours. Il a consulté dans un centre médical local, où on lui a appliqué une compresse qui sentait l'essence. On lui a retiré un « ver » épais de couleur blanche de $2 \mathrm{~cm}$ de long, puis ses symptômes systémiques se sont résorbés sans autre traitement. Une semaine plus tard, il a quitté le Sénégal à bord d'un vol de rapatriement pour le Canada, pendant la pandémie de maladie à coronavirus 2019.

Le patient faisait de la fièvre au moment du prélèvement sanguin. La formule sanguine complète a révélé une numération leucocytaire normale $\left(9,7 \times 10^{9} / \mathrm{L}\right)$, mais un faible taux de lymphocytes, soit $0,8 \times 10^{9} / \mathrm{L}$ (taux normal 1,2-3,5 $\times 10^{9} / \mathrm{L}$ ); la numération plaquettaire et le taux d'hémoglobine étaient normaux. Le dosage des électrolytes, les épreuves de fonction hépatique et le dosage de la transaminase ont tous donné des résultats dans les limites de la plage normale. Le jeune homme avait un taux de protéine $C$ réactive élevé, soit de $116 \mathrm{mg} / \mathrm{L}$ (taux normal < 3,1 mg/L). Étant

\section{POINTS CLÉS}

- Il faut garder à l'esprit une longue liste de diagnostics différentiels en présence d'un voyageur qui revient au pays avec de la fièvre. Afin d'orienter le diagnostic, il faut faire un bilan détaillé des endroits que le patient a visités, des gens qu'il a côtoyés et de ces antécédents d'exposition.

- Les spirochètes observés dans les frottis de sang périphérique habituels ou les frottis servant au dépistage du paludisme sont spécifiques à la fièvre récurrente à tiques ou à poux causée par une bactéries de genre Borrelia.

- En Amérique du Nord, la fièvre récurrente à tiques est endémique en Colombie-Britannique et dans l'ouest des États-Unis, et elle est transmise par les tiques molles de genre Ornithodoros.

- Quelle que soit le type de fièvre récurrente, il faut surveiller étroitement l'apparition d'une réaction de Jarisch-Herxheimer après l'instauration d'un traitement efficace.

donné les antécédents de voyage du patient et sa fièvre récurrente, nous avons placé le paludisme au sommet de notre liste de diagnostics différentiels, tout comme l'infection par le coronavirus du syndrome respiratoire aigu sévère 2 (SRAS-CoV-2). Nous avons effectué un prélèvement nasopharyngé pour le dépistage du SRAS-CoV-2 et demandé un test urgent de dépistage du paludisme. Dans nos établissements régionaux, le dépistage du paludisme est effectué au moyen d'un test antigénique rapide et d'un dépistage préliminaire sur frottis après coloration de Giemsa. Si ces analyses sont négatives, les échantillons sont acheminés vers un laboratoire central, qui effectue une épreuve d'amplification en chaîne par polymérase (PCR) et un examen approfondi des frottis sanguins et gouttes épaisses. Dans ce cas-ci, le test antigénique rapide s'est révélé négatif, et aucun parasite du paludisme n'a été observé sur les frottis minces. L'urgentologue a cependant reçu, plus tard, un appel du laboratoire indiquant la présence de spirochètes (figure 1 ).

Le patient a été orienté vers une clinique d'infectiologie à accès rapide pour la suite du traitement. Comme ils correspondaient au tableau clinique et aux antécédents d'exposition, les spirochètes observés au microscope étaient suffisamment spécifiques pour 


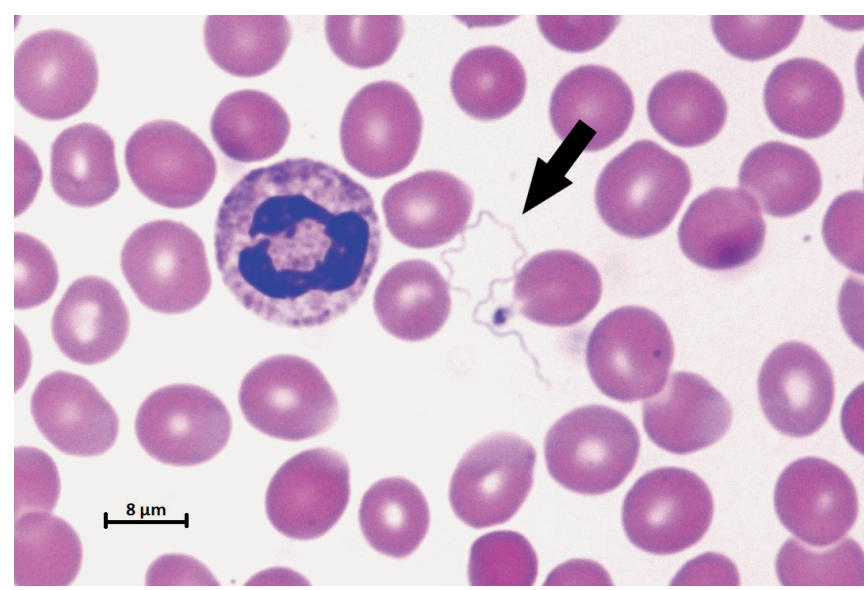

Figure 1 : Frottis de sang périphérique montrant un spirochète isolé.

guider le traitement de la fièvre récurrente. Nous avons administré une dose de ceftriaxone et surveillé le patient pendant 6 heures; nous n'avons observé aucune réaction de Jarisch-Herxheimer. Le lendemain, nous sommes passés à une antibiothérapie de 10 jours à la doxycycline. Lors du suivi initial, 3 jours après le début du traitement, le patient se sentait beaucoup mieux. Le frottis sanguin périphérique effectué à ce moment était négatif pour la présence de spirochètes.

Les bactéries spirochètes, dont le nom est dérivé de leur structure particulière en spirale, comptent plusieurs genres qui sont pathogènes pour l'être humain (encadré 1 ). Après consultation avec le microbiologiste clinique, le British Columbia Centre for Disease Control a procédé à des analyses sérologiques et des tests de PCR et de séquençage pour confirmer le diagnostic. L'immunodosage enzymatique pour Borrelia burgdorferi était positif, mais le test de contrôle par transfert de western était négatif. Le dosage par immunofluorescence pour $B$. hermsii était réactif pour l'immunoglobuline $G(1: 256)$ et non réactif pour l'immunoglobuline M. La recherche de Borrelia par PCR a confirmé la présence de bactéries de ce genre dans le sang. Le séquençage de l'ARNr 1d6S a montré qu'elles étaient étroitement apparentées à $B$. crocidurae (endémique en Afrique de l'Ouest) ou à $B$. hispanica (endémique en Afrique du Nord et autour du bassin

Encadré 1 : Spirochètes importants en médecine

\begin{tabular}{ll} 
Genre & \multicolumn{1}{c}{ Syndrome(s) } \\
Borrelia & - Borréliose de Lyme \\
& - Fièvre récurrente à tiques \\
& - Fièvre récurrente à poux \\
& - Fièvre récurrente à tiques dures (infection à \\
& B. miyamotoi) \\
Treponema & - Syphilis (transmise sexuellement) \\
& - Béjel (syphilis endémique) \\
& - Pian \\
Leptospira & - Pinta \\
Brachyspira & - Spirochétose intestinale
\end{tabular}

méditerranéen). Une fois la borréliose confirmée par le laboratoire, nous avons conclu que le "ver » retiré de la jambe du patient était probablement une larve de mouche (myase) sans lien avec les symptômes systémiques.

\section{Discussion}

\section{Fièvre récurrente}

La fièvre récurrente est une affection distincte caractérisée par des épisodes répétés de fièvre et de symptômes non spécifiques pouvant inclure céphalées, myalgie, arthralgie, frissons intenses et maux de ventre ${ }^{1,2}$. Elle résulte d'une infection par les spirochètes d'une espèce de genre Borrelia, transmis par les poux ou les tiques. Ses complications sont dues à une atteinte du cœur, des poumons, du système nerveux central, du foie, de la rate, des poumons, du tractus gastro-intestinal et des yeux ${ }^{1}$. Il faut distinguer la fièvre récurrente des autres causes d'épisodes fébriles récurrents; l'encadré 2 résume les maladies infectieuses qui peuvent avoir les épisodes de fièvre récurrents parmi leurs principaux symptômes. Une fois l'infection de l'hôte enclenchée, les espèces de genre Borrelia sont capables de modifier leurs antigènes de surface, ce qui entraîne des cycles de présence de spirochètes dans le sang et de stimulation du système immunitaire ${ }^{1}$. La période d'incubation initiale est de 7 jours (intervalle 2-14 jours), suivie d'un premier épisode de fièvre et de symptômes associés d'une durée approximative de 3-5 jours ${ }^{4}$. L'intervalle moyen entre la résolution du premier épisode et la récurrence de la fièvre est $7-9$ jours ${ }^{1}$. Les patients peuvent se sentir tout à fait bien entre les épisodes fébriles, mais parfois les symptômes, comme la sensation de malaise, perdurent ${ }^{1}$.

Il existe 3 formes de fièvre récurrente, chacune définie par son vecteur (encadré 3). La fièvre récurrente à poux résulte d'une infection par l'espèce Borrelia recurrentis, dont la transmission à l'être humain se fait par le pou ${ }^{5}$. Elle se caractérise par une récurrence ou plus, et elle a tendance à évoluer de manière plus violente que les autres fièvres récurrentes. Elle est associée à un taux de mortalité de 10\%-40\% lorsqu'elle n'est pas traitée, et de 2\%-4\% lorsqu'elle est traitée. Elle demeure un problème de santé publique en Afrique du Nord et de l'Est, et pour les populations vivant dans des conditions propices aux infestations de poux $x^{5}$. Contrairement à la fièvre récurrente à poux, la fièvre récurrente à tiques est causée par plus de 15 espèces de Borrelia et est transmise par des tiques molles. Elle peut engendrer plus de 30 épisodes fébriles si elle n'est pas traitée ${ }^{3,4}$. Avec un traitement efficace, le taux de mortalité demeure inférieur à $1 \%$. La Colombie-Britannique est la seule région du Canada où la fièvre récurrente à tiques est endémique; 19 patients ont montré des signes d'infection à $B$. hermsii entre 2006 et $2015^{6}$. Aux États-Unis, cette fièvre s'observe dans les états occidentaux, principalement la Californie, l'État de Washington et le Colorado. Entre 1990 et 2011, 483 cas ont été déclarés dans l'ouest des ÉtatsUnis, la plupart au cours des mois d'été?. Elle peut également se transmettre par des tiques dures; le responsable de la fièvre récurrente à tiques dures, $B$. miyamotoi, a été isolé dans des tiques Ixodes scapularis, identifiées lors d'une surveillance passive partout au Canada ${ }^{8}$. Au Manitoba, des preuves sérologiques d'infection à B. miyamotoi ont été relevées chez des patients que l'on sait ou que l'on croit atteints de la maladie de Lyme'. 
Encadré 2 : Diagnostic différentiel des fièvres récurrentes ${ }^{1-3}$

\section{Maladie}

Agents pathogènes

Zones d'endémicité

Causée par un virus

Fièvre à tiques du Colorado

Fièvre jaune

Dengue

Fièvres hémorragiques africaines

Chorioméningite lymphocytaire

Hépatites virales

Causée par une bactérie

Brucellose

Leptospirose

Sodoku

Bartonellose

Fièvre typhoïde

Ehrlichiose

Rickettsiose

Tularémie

Méningococcémie

Causée par un protozoaire

Paludisme

Babésiose

Leishmaniose viscérale (kala-azar)
Virus de la fièvre à tiques du Colorado

Virus de la fièvre jaune

Virus de la dengue

Virus de Lassa, virus de Marburg, virus Ebola, etc.

Mammarénavirus de la chorioméningite lymphocytaire

Virus des hépatites $A-E$

Genre Brucella

Genre Leptospira

Streptobacillus moniliformis, Spirillum minus

Salmonella enterica, sérovar Typhi ou Paratyphi

Genre Ehrlichia

Genre Rickettsia

Francisella tularensis

Neisseria meningitidis

Genre Plasmodium

Genre Babesia

Genre Leishmania
Genre Bartonella
Amérique du Nord

Amérique du Sud, Afrique

Asie du Sud ou du Sud-Est, Amérique du Sud ou centrale, Caraïbes, Afrique

Afrique

Répandu

Répandu

Répandu

Répandu, surtout dans les régions tropicales ou subtropicales

Surtout Amérique du Nord, Asie

Répandu

Surtout Asie du Sud ou du Sud-Est, sud de l'Afrique Amérique du Nord

Répandu

Amérique du Nord, Europe, Asie

Répandu, mais surtout Afrique subsaharienne (« ceinture de la méningite »)

Afrique, Asie, Amérique du Sud

Amérique du Nord, Europe, Asie

Afrique, Asie, Amérique du Sud ou centrale, Europe (bassin méditerranéen)

\section{Encadré 3 : Comparaison des syndromes de fièvre récurrente}

\begin{tabular}{|c|c|c|c|c|}
\hline Syndrome & Agent pathogène & Vecteur & Hôte réservoir & $\begin{array}{l}\text { Distribution } \\
\text { géographique }\end{array}$ \\
\hline Fièvre récurrente à tiques & $\begin{array}{l}>15 \text { espèces de genre } \\
\text { Borrelia }\end{array}$ & $\begin{array}{l}\text { Tique molle } \\
\text { (genre Ornithodoros) }\end{array}$ & Rongeurs & Monde entier \\
\hline Fièvre récurrente à poux & Borrelia recurrentis & $\begin{array}{l}\text { Pou de corps } \\
\text { (Pediculus humanus } \\
\text { humanus) }\end{array}$ & Humains & $\begin{array}{l}\text { Monde entier (surtout } \\
\text { Afrique de l'Est) }\end{array}$ \\
\hline $\begin{array}{l}\text { Fièvre récurrente à tiques } \\
\text { dures }\end{array}$ & Borrelia miyamotoi & $\begin{array}{l}\text { Tique dure } \\
\text { (genre Ixodes) }\end{array}$ & Rongeurs & Hémisphère nord \\
\hline
\end{tabular}

\section{Diagnostic}

La détection de spirochètes sur des frottis de sang périphérique est fortement indicatrice d'une fièvre récurrente, même si dans de rares cas, on a trouvé d'autres spirochètes sur des frottis après coloration de Giemsa. La densité doit atteindre au moins $10^{4}-10^{5}$ spirochètes par millilitre de sang, ce qui limite la sensibilité du test. La concentration est maximale lorsque l'échantillon est prélevé durant un épisode fébrile ${ }^{1}$. Les frottis de gouttes de sang épaisses, comme ceux demandés pour le diagnostic du paludisme, peuvent accroître la sensibilité du test ${ }^{4}$. Même si plusieurs autres spirochétoses peuvent provoquer une maladie aiguë, comme la borréliose de Lyme et la syphilis, les organismes en cause ne seraient pas faciles à voir, en microscopie optique standard, dans un frottis sanguin ayant subi une coloration de Wright ou de Giemsa; la visualisation de ces spirochètes requiert une immunofluorescence ou une microscopie à fond noir ${ }^{1}$. 
Il est important de reconnaître que la sensibilité des frottis sanguins pour le diagnostic de la fièvre récurrente est limitée comparativement à celle du dosage moléculaire de Borrelia; par conséquent, les professionnels de la santé ne devraient pas se fier aux laboratoires pour identifier d'office Borrelia sans demander d'analyse sérologique, comme ce fut le cas ici. Les analyses sérologiques peuvent être effectuées par immunofluorescence, et un titre de 1:256 ou plus est considéré positif ${ }^{1,6}$. L'épreuve d'amplification en chaîne par polymérase fait partie des tests réalisés par le British Columbia Centre for Disease Control. Le séquençage du génome permet l'identification de l'espèce, un service offert par le Laboratoire national de microbiologie (Winnipeg) ou tout autre laboratoire capable d'effectuer le séquençage de l'ARNr 16S sur des échantillons cliniques.

\section{Traitement}

Il est recommandé de traiter la fièvre récurrente à poux chez les adultes, à l'exception des femmes enceintes, avec une dose unique de $100 \mathrm{mg}$ de doxycycline ou de $500 \mathrm{mg}$ de tétracycline. Sinon, une dose simple d'érythromycine $(500 \mathrm{mg}$ ) ou de pénicilline $\mathrm{G}$ procaïne intramusculaire $(600000 \mathrm{U})$ est efficace ${ }^{1,2}$. La fièvre récurrente à tiques requiert la prise de doxycycline (100 mg, 2 fois par jour) ou d'érythromycine (500 mg, 4 fois par jour) pendant $7-10$ jours $^{1,2}$. Chez les patients qui sont incapables de prendre des médicaments oraux ou qui ont une atteinte du système nerveux central, un traitement parentéral à la ceftriaxone $(2 \mathrm{~g} / \mathrm{jour})$ ou à la pénicilline $\mathrm{G}$ (injection intraveineuse de 3 millions d'unités toutes les 4 heures) pourrait être nécessaire ${ }^{2}$. Après la première dose de tout antibiotique efficace, il faut rester à l'affût de la réaction de Jarisch-Herxheimer, qui survient habituellement dans les 4 premières heures ${ }^{1,2}$. Cette réaction potentiellement mortelle est causée par la libération d'endotoxines due à la destruction rapide des spirochètes, ce qui crée un choc cytokinique. Les symptômes peuvent inclure fièvre, frissons intenses, tachycardie, diaphorèse, hypotension et détresse respiratoire ${ }^{1,6}$.

\section{Conclusion}

La bactérie de genre Borrelia causant la fièvre récurrente de notre patient a été identifiée de façon fortuite par un technologiste de laboratoire, après un protocole diagnostique pour le paludisme. Selon les lignes directrices canadiennes ${ }^{10}$, il faut d'abord écarter un diagnostic de paludisme chez les patients fébriles qui ont voyagé dans des régions où la maladie est endémique; toutefois, les médecins devraient aussi soupçonner une fièvre récurrente et demander les analyses sérologiques et moléculaires pertinentes selon le cas.

\section{Références}

1. Dworkin MS, Schwan TG, Anderson DE, et al. Tick-borne relapsing fever. Infect Dis Clin North Am 2008;22:449-68.

2. Cutler SJ. Relapsing fever Borreliae: a global review. Clin Lab Med 2015;35:847-65.

3. Thwaites GE, Day NPJ. Approach to fever in the returning traveler. N Engl J Med 2017;376:1798

4. Southern PMJ, Sanford JP. Relapsing fever: a clinical and microbiological review. Medicine (Baltimore) 1969;48:129-50.

5. Badiaga S, Brouqui P. Human louse-transmitted infectious diseases. Clin Microbiol Infect 2012;18:332-7.
6. Morshed MG, Drews SJ, Lee M-K, et al. Tick-borne relapsing fever in British Columbia: a 10-year review (2006-2015). B C Med J 2017;59:412-7.

7. Tick-borne relapsing fever - distribution. Atlanta: Centers for Disease Control and Prevention; 2015. Accessible ici : www.cdc.gov/relapsing-fever/distribution/ index.html (consulté le 8 déc. 2020).

8. Dibernardo A, Cote $\mathrm{T}$, Ogden $\mathrm{NH}$, et al. The prevalence of Borrelia miyamotoi infection, and co-infections with other Borrelia spp. in Ixodes scapularis ticks collected in Canada. Parasit Vectors 2014;7:183.

9. Kadkhoda K, Dumouchel C, Brancato J, et al. Human seroprevalence of Borrelia miyamotoi in Manitoba, Canada, in 2011-2014: a cross-sectional study. CMAJ Open 2017;5:E690-3.

10. Chapter 6 - Malaria diagnosis: Canadian recommendations for the prevention and treatment of malaria. Ottawa: Public Health Agency of Canada; [updated 2020 Jan. 16]. Accessible ici : www.canada.ca/en/public-health/services/catmat/ canadian-recommendations-prevention-treatment-malaria/chapter-6-malaria -diagnosis.html (consulté le 8 déc. 2020).

Intérêts concurrents : Aucun intérêt concurrent déclaré.

Cet article a été révisé par des pairs.

Les auteurs ont obtenu le consentement du patient.

Affiliations : Division de microbiologie médicale et de lutte contre les infections (Eckbo, Charles), Hôpital général de Vancouver; Départements de pathologie et médecine de laboratoire (Eckbo, Charles, Wolber) et de médecine (Yu), Université de la Colombie-Britannique; Département de pathologie et médecine de laboratoire (Wolber) et Division de l'infectiologie (Yu), Hôpital Lions Gate, Vancouver, C.-B.

Collaborateurs : Tous les auteurs ont contribué à l'élaboration et à la conception des travaux. Eric Eckbo et Robert Wolber ont recueilli les données. Eric Eckbo a rédigé l'ébauche du manuscrit. Tous les auteurs ont révisé de façon critique le contenu intellectuel important du manuscrit; ils ont donné leur approbation finale pour la version destinée à être publiée et assument l'entière responsabilité de tous les aspects du travail.

Propriété intellectuelle du contenu : Il s'agit d'un article en libre accès distribué conformément aux modalités de la licence Creative Commons Attributions (CC BY-NC-ND 4.0), qui permet l'utilisation, la diffusion et la reproduction dans tout médium à la condition que la publication originale soit adéquatement citée, que l'utilisation se fasse à des fins non commerciales (c.-à-d., recherche ou éducation) et qu'aucune modification ni adaptation n'y soit apportée. Voir : https://creativecommons.org/licenses/by-nc-nd/4.0/deed.fr.

Remerciements : Les auteurs remercient le Dr Muhammad Morshed et Min-Kuang Lee du British Columbia Centre for Disease Control pour leur aide dans la collecte et l'interprétation des données, les examens diagnostiques approfondis effectués chez le patient, leur expertise en ce qui concerne les infections à spirochètes et la révision du manuscrit.

Correspondance : Eric Eckbo, eric.eckbo@vch.ca

La section Études de cas présente de brefs rapports de cas à partir desquels des leçons claires et pratiques peuvent être tirées. Les rapports portant sur des cas typiques de problèmes importants, mais rares ou sur des cas atypiques importants de problèmes courants sont privilégiés. Chaque article commence par la présentation du cas (500 mots maximum), laquelle est suivie d'une discussion sur l'affection sous-jacente (1000 mots maximum). La soumission d'éléments visuels (p. ex., tableaux des diagnostics différentiels, des caractéristiques cliniques ou de la méthode diagnostique) est encouragée. Le consentement des patients doit impérativement être obtenu pour la publication de leur cas. Renseignements destinés aux auteurs : www.cmaj.ca 